of the hospital has been replanted by the Staffordshire trust for nature conservation. ${ }^{5}$

Inner city hospitals on tiny sites have more difficulties, but older city hospitals with courtyards, such as the Middlesex and St Bartholomew's have continued to encourage patients, staff, and visitors to use their gardens. St George's Hospital, a city hospital built in the 1970 s, has a sculptured courtyard by artist Shelagh Wakely and external gardens, and phase 1 of the new Hammersmith Hospital has a roof courtyard garden with a water sculpture by William Pye, with wheelchair access and low window sills for easy visibility from patients' beds, as well as from the corridor and dayrooms.

Much of the lead in British thinking about hospital gardens has come from the Department of Health, which has emphasised the need for a good quality visual environment in hospitals. In its general introduction to health building, the department accepted advice to state boldly that hospitals should be beautiful as well as functional. A good looking building accompanied by pleasant landscaping improves morale of staff and patients and can be just as economical as an unattractive one. It is to be hoped that the Poor Law mentality which assumes that a barren dreariness befits an NHS building, and that an agreeable environment denotes extrava- gance, is a thing of the past. ${ }^{6}$ More detailed advice on gardens is included in the recent health building note $45 .^{7}$ As a result of such guidance the United Kingdom has taken the lead in showing concern for the patient's environment and is reinstituting the use of gardens to help patients to restore their sense of well being.

SAM BASS WARNER Jr Jack Meyerhoft Professor of American Environmental Studies

Brandeis University,

Waltham, MA 02254-9110, USA

JH BARON

Chairman

British Healthcare Arts,

Duncan of Jordanstone College of Art,

Dundee DD1 4H7

1 Malkin J. Hospital interior architecture. New York: Van Nostrand Reinhold, 1992;13-37.

2 Ulrich RS, Parsons R. Influences of passive experiences with plants on individual well being and health. In: Relf $\mathrm{D}$, ed. The rose of horticulture in human well-being and social development. (Portland, Oregon: Timber Press, 1992:93-105.

3 Howard J. An account of the principal lazarettos in Europe ... together with further observation on some foreign prisons and hospitals etc. Warrington: William Eyres, 1789.

4 Taylor JRB. Hospital and asylum architecture in England 1840-1914. London: Mansell Publishing, 1991:58-60, 81-3, 144-5, 150-1.

5 Boddington J. Turning Japanese. Hospital development 1990;1:37-40.

6 Department of Health. Buildings for the health service. London: HMSO, 1988 (Health Building Note 1).

7 NHS Estates. External works for Health Buildings. London: HMSO, 1992 (Health Building Note 45)

\title{
A role for medical angiologists?
}

\author{
Firstly, they will have to prove their worth
}

The European Working Group on Medical Angiology wants to develop the specialty of medical angiology. ${ }^{1}$ What is this specialty, do we need it, and, crucially, will purchasers purchase it in the reformed NHS?

No one would dispute with the working group that disorders of the circulation, venous as well as arterial, are common, costly, and a burden on society. But this is not enough to justify a new medical specialty encompassing all diseases of the vascular system, irrespective of the organ affected, unless it improves the care of patients with coronary heart disease, stroke, peripheral vascular disease, and venous thromboembolism. Let us therefore consider these disorders in turn, at least from a British perspective, although I don't doubt the eventual need for a European perspective in all specialties as well as some agreement about what particular specialties are supposed to do.

The biggest impact on the prevention of arterial disease is likely to come from political action to reduce cigarette smoking, improve nutrition, and facilitate exercise in the whole population. ${ }^{2}$ High risk patients (for example, those with angina, diabetes, and hypertension) usually have their vascular risk factors dealt with in general practice, where there is no direct role for a medical angiologist. Angiologists could, however, facilitate preventive strategies both in the community at large and in general practice. Such specialists might also provide clinics for particularly high risk patients in hospitals where none presently exists, although any impact on the incidence of disease would be small because most cases arise in patients who are at moderate risk. ${ }^{2}$ Nonetheless, some patients would benefit.

The treatment of coronary heart disease is already organised by cardiologists and cardiac surgeons; much clinical and laboratory research is being done; and any improvement would almost certainly be seen in terms of increasing the numbers in these clinical specialties rather than introducing a new one. Of course, most symptomatic patients get most help from general physicians and general practitioners, who refer them to specialised cardiac services when necessary, and this seems to work well. Cardiac units already provide imaging facilities and angioplasty.

Although stroke is treated haphazardly, ${ }^{3}$ many efforts are being made to improve this. ${ }^{45}$ Patients with stroke or transient ischaemic attack are probably best managed by neurologists with occasional help from vascular surgeons and neurosurgeons, as occurs in the Netherlands. Neurologists should be the best people to manage what is, after all, the most common serious neurological disorder, but there are not enough to do so in Britain and, perversely, many are not interested in doing so. In any event, British neurologists do not have adequate access to rehabilitation, day hospitals, and facilities for respite and long term care. On the other hand, geriatricians do have these facilities so that a comprehensive stroke service in Britain should probably be provided by a partnership between geriatricians and interested neurologists. No need for medical angiologists here.

At present, the management of peripheral vascular disease is almost completely in the hands of surgeons; they have no "medical" partners. Could physicians with a special interest in disorders of the peripheral vascular system, medical angiologists perhaps, help? Probably yes. They could take on the initial diagnostic work up and sort out the myriad of neurological, orthopaedic, rheumatological, psychological, and other causes of pain in the leg; orchestrate the medical management of peripheral vascular disease, which entails much more than just caring for the leg arteries; and bring in the vascular surgeons for the few patients who need surgery (very much in the way that neurologists work with vascular surgeons in the management of transient ischaemic attack). As a result, surgeons would be able to spend more time developing their surgical skills and techniques. Physicians would also, I suspect, be a little more forward than surgeons 
in organising the randomised trials that are needed to evaluate the numerous interventions that have been suggested and are in current use.

Finally, venous thromboembolism. Deep vein thrombosis and pulmonary embolism are avoidable and treatable risks for all ill patients. Yet few hospitals provide a coherent and informed preventive service, or a good treatment service, of the sort that has been pioneered at McMaster University Medical School in Ontario. There is surely a gap here which medical angiologists could fill, because both prevention and treatment are almost entirely medical rather than surgical, much is known. and can be done, and certainly more research is needed.

There is therefore a good argument to develop medical services for patients with peripheral vascular disease in collaboration with vascular surgeons and services for venous thromboembolism. Physicians with an interest in such disorders-medical angiologists-might also take on Raynaud's disease and other vasospastic disorders, perhaps contribute to vascular prevention clinics, and possibly plug gaps in services for coronary heart disease and stroke. But they cannot spread themselves too thinly because to cover all vascular disorders would require training and competence in general medicine, cardiology, neurology, and haematology -which would be completely unrealistic.

At present there are perhaps five physicians in Britain who would admit to being medical angiologists, though I suspect that they are counted as general physicians. The European working group's proposal for one or two per 100000 population is so unrealistic that it undermines the group's case. It would mean as many as 1000 medical angiologists in Britain. To start with it would be best to train and appoint some general physicians with an interest in angiology in a few teaching hospitals and large district general hospitals. Eventually, however, medical angiologists would have to convince purchasers to buy their services-so someone somewhere needs to show that the outcome for patients with peripheral vascular disease would be improved and that the incidence and ravages of venous thromboembolism could be reduced. Here, then, is the challenge to medical angiologists: prove that we need you.

C P WARLOW

Department of Clinical Neurosciences, Professor of medical neurology

University of Edinburgh,

Western General Hospital,

Edinburgh EH4 2XU

1 European Working Group on Medical Angiology. The case for the speciality of medical angiology Angiol 1991;10:199-201.

2 Rose G. The strategy of preventive medicine. Oxford: Oxford Medical Publication, 1992

3 King's Fund. Treatment of stroke. BMF 1988;297:126-8.

4 Royal College of Physicians of London. Stroke: towards better management. London: RCP, 1989.

5 Stroke rehabilitation. Effective health care 1992; No 2.

\section{Mental health problems at work}

\section{Cause thirty times as many lost days as industrial disputes}

The amount of time lost from work through mental health problems is enormous-in England and Wales it amounts to 80 million working days a year, costing employers $£ 3.7$ billion, according to the then secretary of state for health, Mr William Waldegrave, at a conference in 1991. A more comprehensive estimate from the Confederation of British Industry came to $£ 5.3$ billion. ${ }^{1}$ This is 30 times the number of days lost through industrial disputes, and the figures are probably an underestimate as self certification is excluded and mental health problems may be disguised by other diagnostic terms.

Most people attribute the cause of their mental health problems to their job. ${ }^{23} \mathrm{~A}$ direct correlation between stress at work and emotional symptoms suggests that they may be right, but a critical examination must include consideration of other factors, including personal susceptibility and stress outside work.

Suicide rates associated with certain occupations have been compared, but these are a crude measure and probably unreliable because standardised mortality ratios are based on tiny numbers. Emotional distress detected by self administered questionnaires, such as the general health questionnaire or the Crown Crisp experiential index, indicate higher rates in people in occupations that might be considered to be more stressful: student nurses, teachers, social workers, and junior medical staff. ${ }^{45}$

Despite the common myth that executives suffer most stress, those in lower employment grades report lower job satisfaction, less varied work, and less control over their work. These factors concur with the occupational psychology literature that characterises job stress as irregular workloads, lack of career prospects, and a feeling of being undervalued and of capabilities not being used. ${ }^{5}$ Using rates adjusted for the tendency of lower employment grades to "underreport" symptoms on the general health questionnaire ${ }^{26}$ the Whitehall study showed a significantly higher rate of psychiatric morbidity in the lower employment grades for men but not for women. ${ }^{6}$ This gender difference might be explained by the greater social support of women in lower grades. $^{7}$ Alternatively, women managers may have to show a particularly high degree of type A behaviour to succeed, resulting in greater symptoms of stress in women of higher grades. ${ }^{8}$

There has been inadequate research relating personality and other variables to stress at work; low self reported job satisfaction may simply reflect neuroticism or current depression. Studies of back pain and heart disease have touched on the relation. In a longitudinal study of aircraft employees personality was related to back problems but the single best predictor of future back injury, even when sex and previous back problems, were controlled for, was job (dis)satisfaction..$^{10}$ Studies of type A personality have shown, on the one hand, that companies with profitable sales trends and a higher return on investment employ more people with type A personalities. On the other hand, type A personality in conjunction with long working hours, few holidays, and other work problems is associated with subsequent myocardial infarction. ${ }^{11}$

Factors outside work may also be important. Those in lower grades in the Whitehall study reported more adverse social circumstances and more stressful life events than those in higher grades. ${ }^{7}$ The relative contributions of work and home related stressors to depression were assessed in a professional and managerial cohort in the United States, of 